\title{
The magnetic fields of hot subdwarf stars ${ }^{\star}$
}

\author{
J. D. Landstreet ${ }^{1,2}$, S. Bagnulo ${ }^{1}$, L. Fossati ${ }^{3}$, S. Jordan ${ }^{4}$, and S. J. O'Toole ${ }^{5}$ \\ 1 Armagh Observatory, College Hill, Armagh, BT61 9DG, Northern Ireland, UK \\ e-mail: [jls; sba]@arm.ac.uk \\ 2 Department of Physics \& Astronomy, University of Western Ontario, London, ON N6A 3K7, Canada \\ e-mail: jlandstr@uwo.ca \\ 3 The Open University, Walton Hall, Milton Keynes, MK7 6AA, UK \\ e-mail: 1. fossati@open.ac.uk \\ ${ }^{4}$ Astronomisches Rechen-Institut, Zentrum für Astronomie der Universität Heidelberg, Mönchhofstr. 12-14, 69120 Heidelberg, \\ Germany \\ e-mail: jordan@ari.uni-heidelberg.de \\ 5 Australian Astronomical Observatory, PO Box 296, 1710 Epping NSW, Australia \\ e-mail: otoole@aao.gov.au
}

Received 6 March 2012 / Accepted 29 March 2012

\begin{abstract}
Context. Detection of magnetic fields has been reported in several sdO and sdB stars. Recent literature has cast doubts on the reliability of most of these detections. The situation concerning the occurrence and frequency of magnetic fields in hot subdwarfs is at best confused.

Aims. We revisit data previously published in the literature, and we present new observations to clarify the question of how common magnetic fields are in subdwarf stars.

Methods. We consider a sample of about 40 hot subdwarf stars. About 30 of them have been observed with the FORS1 and FORS2 instruments of the ESO VLT. Results have been published for only about half of the hot subdwarfs observed with FORS. Here we present new FORS1 field measurements for 17 stars, 14 of which have never been observed for magnetic fields before. We also critically review the measurements already published in the literature, and in particular we try to explain why previous papers based on the same FORS1 data have reported contradictory results.

Results. All new and re-reduced measurements obtained with FORS1 are shown to be consistent with non-detection of magnetic fields. We explain previous spurious field detections from data obtained with FORS1 as due to a non-optimal method of wavelength calibration. Field detections in other surveys are found to be uncertain or doubtful, and certainly in need of confirmation.

Conclusions. There is presently no strong evidence for the occurrence of a magnetic field in any sdB or sdO star, with typical longitudinal field uncertainties of the order of 2-400 G. It appears that globally simple fields of more than about 1 or $2 \mathrm{kG}$ in strength occur in at most a few percent of hot subdwarfs. Further high-precision surveys, both with high-resolution spectropolarimeters and with instruments similar to FORS1 on large telescopes, would be very valuable.
\end{abstract}

Key words. subdwarfs - stars: magnetic field - magnetic fields

\section{Introduction}

Hot subdwarf ( $\mathrm{sdB}$ and $\mathrm{sdO}$ ) stars are subluminous relative to main sequence stars of the same effective temperature. They are the most common type of faint blue star in the galaxy.

The sdB stars have effective temperature $T_{\text {eff }} \lesssim 40000 \mathrm{~K}$, and generally have H-rich atmospheres. They are mostly deficient in atmospheric He, and often in several other light elements such as $\mathrm{C}, \mathrm{O}, \mathrm{Mg}$ and $\mathrm{Al}$. A few of them show greatly enriched iron peak element abundances. The sdO stars have a large range of $\mathrm{H} / \mathrm{He}$ abundance ratios, and show a variety of compositions. Their $T_{\text {eff }}$ values mostly lie between 40000 and $90000 \mathrm{~K}$.

Both $\mathrm{sdB}$ and sdO stars have lost most or all of their H-rich outer envelopes, due to mass loss and/or binary mass transfer, and are now burning $\mathrm{He}$ in their cores. In turn, a small fraction of white dwarfs will descend from them. However, the details of

^ Based on observations collected at the European Organisation for Astronomical Research in the Southern Hemisphere, Chile under observing programmes 072.D-0290 and 075.D-0352, or obtained from the ESO/ST-ECF Science Archive Facility. how stars evolve to become hot subdwarfs, and particularly how such a wide variety of atmospheric chemistries is produced, are very poorly understood.

Because many hot subdwarfs show strong atmospheric composition anomalies, which on the main sequence are frequently found in magnetic Ap-Bp stars, it is natural to wonder if some or all hot subdwarfs also possess detectable magnetic fields. The presence or absence of a field could be an underlying parameter influencing the observed surface composition, as in magnetic upper main sequence stars. If fields can be detected in hot subdwarfs, these stars could complement the main sequence magnetic stars as laboratories in which to study the operation of such processes as atomic diffusion, surface convection, internal mixing, and mass loss in the presence of global magnetic fields (Landstreet 2004).

More generally, strong global fields are known to occur in some hot stars (i.e. stars without deep outer convection zones and active current magnetic dynamos) on the main sequence and in the white dwarf state. Hot subdwarfs represent a possible intermediate stage between the main sequence and the collapsed 
white dwarf state, in which the interior of the former main sequence star is exposed to observation, and again lacks deep outer convection. Detection of a magnetic field in any hot subdwarf could be very helpful to understanding the evolution of a global internal magnetic field as the host star changes in structure due to stellar evolution. Even clear evidence that fields are not present in hot subdwarfs down to some small upper limit provides a useful constraint on theory.

For these reasons, several surveys have been conducted to search for magnetic fields in $\mathrm{sdB}$ and $\mathrm{sdO}$ stars. At first, it seemed that these surveys were very successful in detecting kG-strength fields in a number of hot subdwarfs (Elkin 1996; O'Toole et al. 2005), but recent work by Petit et al. (2012) and Bagnulo et al. (2012), has raised doubts about the reliability of most of the detections. A recent survey of cool sdB stars by Mathys et al. (2012), based on FORS2 data, reported a suspected field in only one out of ten $\mathrm{sdB}$ stars. Currently the situation is quite confused; it is not clear if magnetic fields are common among hot subdwarfs, or rare, or even if any have been detected at all. If any hot subdwarf fields have in fact been detected, almost nothing is known about the characteristics of the fields.

A first step is to clearly establish what is known from previous work in this field. The goal of this paper is to clarify the present situation, to establish which field detections (if any) are robust, and which are doubtful. To do this, we review the previous surveys, expanding on work already presented by Bagnulo et al. (2012). Furthermore, we publish for the first time further field measurements of 17 hot subdwarfs carried out with FORS1. Based on a sample of 41 stars, we finally draw conclusions on the incidence of magnetic fields in hot subdwarfs.

\section{Previous magnetic measurements}

A number of searches for magnetic fields in hot subdwarf stars have been carried out, for a total (to the best of our knowledge) of 25 objects surveyed.

Borra et al. (1983) used a Cassegrain filter polarimeter equipped with narrow band interference filter to measure the circular polarisation in the wings of $\mathrm{H} \beta$. They were able to obtain one field measurement each of Feige $86=\mathrm{BD}+302431$ and HD 149382 = BD-03 3967, but because of the faintness of the stars $(V \sim 9-10)$ and the use of only a single spectral line, the standard errors of measurement reported were about $2800 \mathrm{G}$. No significant field was detected to this precision in either star.

Spectropolarimetric observations of sdO stars by Elkin (1996) were obtained using a classical fixed polarisation analyser on the Russian 6-m telescope to measure Zeeman polarisation in the He I D3 line $\lambda$ 5876. Two hot subdwarfs, BD+75 325 and Feige $66=\mathrm{BD}+25$ 2534, were observed, and field detections at the $\mathrm{kG}$ level were reported for both stars. It is difficult to know how secure these detections are. Although Elkin (1996) standardised his measurements each night with observations of both a null and a polarised standard star, repeated field measurements of one of the two hot subdwarfs on a single night could vary by as much as $1 \mathrm{kG}$, which is of the same order as the reported fields. The reported standard errors of $250 \mathrm{G}$ or more for the polarisation standard $(53 \mathrm{Cam})$, with about a dozen sharper spectral lines in the $120 \AA$-wide window used, suggests that the uncertainty of field measurement using only one broader line could be of the order of $1 \mathrm{kG}$. Our view is that these reported detections certainly would need to be confirmed by further observations with a more sensitive method before they could be considered secure.
O'Toole et al. (2005) reported field measurements of six different $\mathrm{sdB}$ and sdO stars ( $T_{\text {eff }}$ between 25000 and $70000 \mathrm{~K}$ ), all obtained during a single night. Each star was observed once using FORS1 in spectropolarimetric mode, and in each of the six stars a field of about $-1 \mathrm{kG}$ was detected, with reported uncertainties of the order of 100-230 G.

Petit et al. (2012) recently published new ESPaDOnS observations of two hot subdwarfs, Feige 66, and HD 76431, in which field detections had been previously reported by Elkin (1996), and by O'Toole et al. (2005) respectively. No fields were detected with the new observations, with $\left\langle B_{z}\right\rangle$ uncertainties of $200 \mathrm{G}$ and $55 \mathrm{G}$, respectively. Furthermore, Petit et al. (2012) re-analysed the old FORS1 data of HD 76431 used by O'Toole et al. (2005), and the revised field measurement was found consistent with zero. In conclusion, the reported field detections for Feige 66 and HD 76431 are both quite uncertain.

All FORS1 measurements by O'Toole et al. (2005) have been re-analysed by Bagnulo et al. (2012). For all six stars, the new reductions failed to confirm the reported fields. It appears to us that the problem with the original reductions by O'Toole et al. (2005) is that separate wavelength calibrations were used for the two waveplate settings of the FORS1 polarimeter, thus effectively sabotaging the possibility of using measurements from the two settings to cancel out first-order errors in the relative wavelength calibration of the two analysed beams. As discussed by Bagnulo et al. (2009), tiny numerical differences in the wavelength calibration of the frames obtained at different position angles of the retarder waveplate may lead to noticeable spurious polarisation signals. In the present case, the residual uncertainty in the different calibrations, of order $0.03 \AA$, is quite large enough to introduce field measurement errors of the order of $1 \mathrm{kG}$, and this might be the reason all six stars observed appear to have very similar longitudinal field strengths. Thus Bagnulo et al. (2012) concluded that the field detections reported by O'Toole et al. (2005) were spurious. A similar problem was discovered for FORS1 observations of central stars of planetary nebulae, leading to revision of previously reported field detections (Jordan et al. 2012).

Three field measurements of the sdO star WD 1036+433 = Feige 34 were reported by Valyavin et al. (2006), using a lowresolution spectropolarimeter at the prime focus of the Russian 6-m telescope. This instrument is conceptually rather similar to FORS1, with a resolving power of $\sim 2000$, and a rotating quarterwave plate in the polarisation analyser, thus making it possible to cancel out errors in the wavelength calibration of the two beams to first order. One of the three observations showed a magnetic field of $\left\langle B_{z}\right\rangle=9.6 \pm 2.6 \mathrm{kG}$, significant at the $3.7 \sigma$ level. The other two measurements were both consistent with zero field, although one was significant at the $2 \sigma$ level. We consider that this field detection may be correct, but because only one measurement is significant, and only at a little more than the $3 \sigma$ level, it certainly requires further confirming observations.

Recently, Savanov et al. (2011) analysed the spectropolarimetric observations of the bright $(B=11 \mathrm{~m} .8)$ non-radially oscillating sdB star Balloon 90100001 = TYC 2248-1751-1 taken with the main stellar spectrograph of the $6 \mathrm{~m}$ Special Astrophysical Observatory. They did not detect any magnetic field, and their measured field value was $34 \pm 63 \mathrm{G}$, considerably lower than the field strengths reported for other hot subdwarfs by O'Toole et al. (2005) and Valyavin et al. (2006).

During the course of a survey of about 60 DA white dwarfs for weak fields, made with the Steward Observatory CCD spectropolarimeter, field measurements were obtained for four sdB stars by Kawka et al. (2007). This survey is remarkable for 
the number of non-detections reported, which strongly confirm that the instrument is not prone to false positive detections. No fields were found in the observations (one or two per star) of the four hot subdwarfs, with reported uncertainties in the range of 4-12 kG.

Finally, a new survey of 10 cool sdB stars using FORS2 (which now has the polarimetric optics formerly installed on FORS1) has just been reported by Mathys et al. (2012). This group standardised their observations with measurements of the magnetic Ap star HD 142070. Each star was observed between two and four times. One observation (of three) of the star SB 290 is significant at about the $4 \sigma$ level, and a second is significant at about the $2.5 \sigma$ level. It is quite possible that a field has been detected in this star, but, as the authors point out, their provisional detection certainly needs to be confirmed by further observations.

What is remarkable about the observations discussed above is the fact that, although apparently significant fields have been reported in a total of 10 hot subdwarfs, new measurements of only two of these stars have been reported (by Petit et al. 2012) which were able to confirm (or fail to confirm, in this specific case) the reality of the reported field detections.

\section{New FORS1 magnetic field measurements}

Polarised spectra of 17 hot subdwarfs were obtained in service mode between March and September 2005 (one star was observed twice) with grism $600 \mathrm{~B}$ and a $0.5^{\prime \prime}$ slit width, for a spectral resolving power between 1400 and 1500. Data were reduced and analysed as described in Bagnulo et al. (2012), with the difference that we have implimented a sigma-clipping process in the determination of the magnetic field from the correlation diagram of circular polarisation against local flux derivative. The effective Landé factor was set to 1 for $\mathrm{H}$ Balmer lines and 1.15 everywhere else. The observing log and results are summarised in Table 1. Successive columns list two star names, spectral type, stellar parameters mostly found from the hot subdwarf database (Østensen et al. 2010, see http: //catserver .ing. iac.es/sddb/searchcat.html), the exposure times and the peak signal-to-noise ratio per $\AA$ in the spectrum, the MJD of mid-observation, and the field strength $\left\langle B_{z}\right\rangle$ and associated standard error in $\mathrm{G}$ as inferred from Stokes $V$. Null profiles (which are representative of the error of the observed Stokes profiles, see, e.g., Bagnulo et al. 2009) were also calculated, and the mean longitudinal field obtained from the null profile was always found consistent with zero within the error bars. A thorough discussion on this kind of diagnostic check is reported in Bagnulo et al. (2012). An example of reduced data is shown in Fig. 1.

Table 1 includes the six field measurements discussed by O'Toole et al. (2005) and revised for the present work. Note that the actual field strengths deduced from the FORS1 data for the stars observed by O'Toole et al. (2005) are slightly (but not significantly) different from the revised estimate published by Bagnulo et al. (2012) because the implementation of the sigmaclipping algorithm has marginally changed the output of our reductions, and because of slightly different choices of the effective Landé factors adopted for He and metal lines.

Two observations of the sdO star CD-22 9142, originally taken for a white dwarf observing project (Jordan et al. 2007), were also included in the table. One additional archive measurements for WD 0958-073 was reduced, but not included in Table 1 because the measurement, although a null, has a standard error of about $5 \mathrm{kG}$.
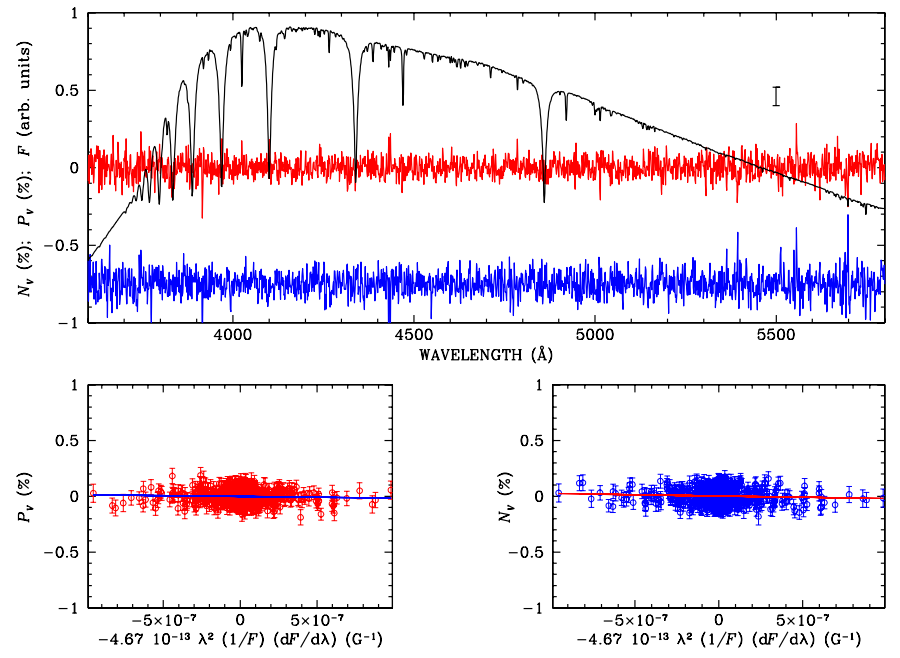

Fig. 1. The observations of HD 205805 obtained with FORS1 on 200506-12. The top panel shows the observed flux $F$ (black solid line, in arbitrary units, and not corrected for the instrument response), the $P_{V}=$ $V / I$ profile (red solid line centred about 0 ), and the null profile $N_{V}$ (blue solid line, offset by $-0.75 \%$ for display purpose). The null profile is expected to be centred about zero and scattered according to a Gaussian with $\sigma$ given by the $P_{V}$ error bars. A typical $P_{V}$ error bar is shown in the upper right of the upper panel. The slope of the interpolating lines in the bottom panels give the mean longitudinal field from $P_{V}$ (left bottom panel) and from the null profile (right bottom panel), both calculated using the $\mathrm{H}$ Balmer and metal lines. The corresponding $\left\langle B_{z}\right\rangle$ and $\left\langle N_{z}\right\rangle$ values are $-139 \pm 120 \mathrm{G}$ and $-220 \pm 140 \mathrm{G}$, respectively.

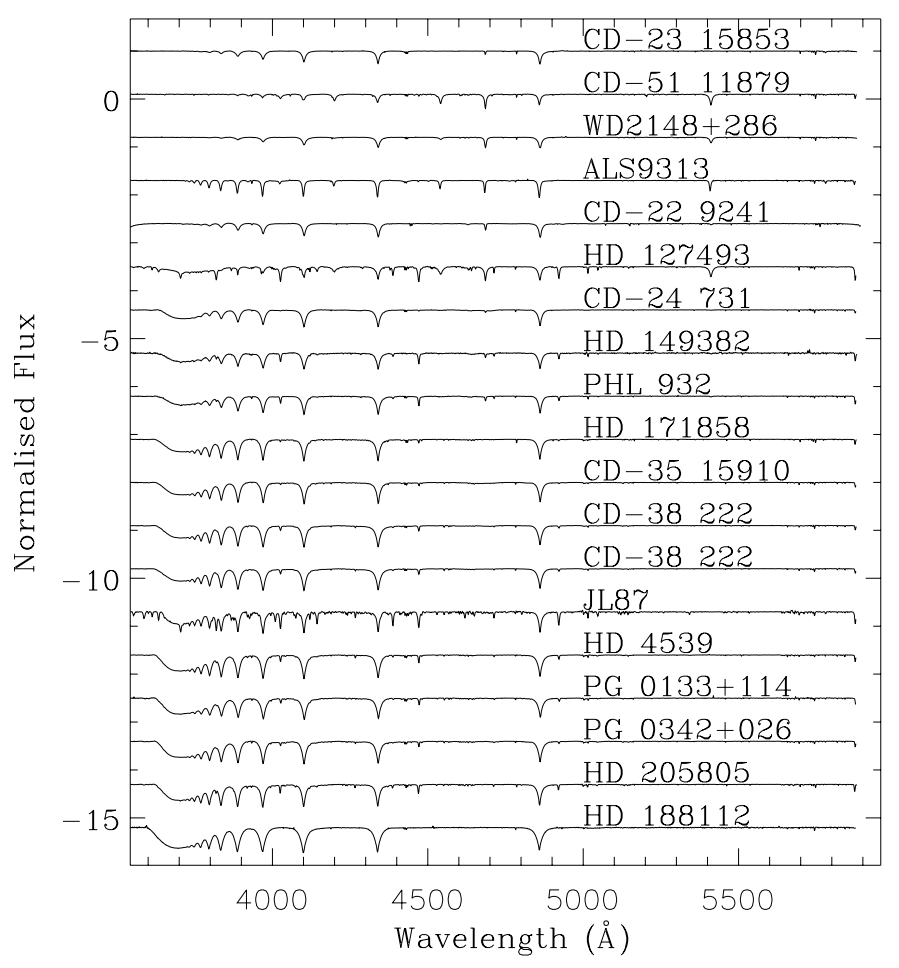

Fig. 2. The normalised fluxes of 18 stars of Table 1 for which new field determinations are presented in this paper.

Figure 2 shows the normalised Stokes $I$ spectra for all newly measured stars of Table 1. 
Table 1. Fundamental stellar parameters and FORS1 magnetic field measurements of hot subdwarf stars.

\begin{tabular}{|c|c|c|c|c|c|c|c|c|c|c|}
\hline Star & & $\begin{array}{l}\text { Spect. } \\
\text { type }\end{array}$ & $\begin{array}{l}T_{\text {eff }} \\
(\mathrm{K})\end{array}$ & $\log g$ & $\begin{array}{l}\text { Exp. time } \\
\text { (s) }\end{array}$ & $\begin{array}{c}\text { Peak } S N R \\
\left(\AA^{-1}\right)\end{array}$ & \multicolumn{4}{|c|}{$\begin{array}{l}\left\langle B_{z}\right\rangle \\
(\mathrm{G})\end{array}$} \\
\hline CD-38222 & SB 290 & $\mathrm{SD}: \mathrm{B}$ & 28200 & 5.5 & 3024 & 1865 & 53574,364 & 32 & +150 & \\
\hline & & & & & 1512 & 1430 & 53624.097 & -172 & \pm 202 & \\
\hline HD 4539 & PG 0044+097 & SD:B & 27000 & 5.5 & 1260 & 1340 & 53058.218 & 554 & \pm 181 & \\
\hline PHL 932 & PG 0057+155 & SD:B & 33650 & 5.7 & 2760 & 960 & 53593.256 & -3 & \pm 307 & \\
\hline PG $0133+144$ & Baloon 92627001 & SD:B & & & 1824 & 775 & 53638.250 & -1074 & \pm 388 & \\
\hline CD-24 731 & SB 707 & SD:B & 37000 & 6.0 & 2700 & 1205 & 53629.135 & 363 & \pm 411 & \\
\hline PG $0342+026$ & WD $0342+026$ & SD:B & 26200 & 5.7 & 2634 & 1570 & 53593.377 & -113 & \pm 172 & \\
\hline HD 127493 & BD-22 3804 & SD:O & 41000 & 5.1 & 984 & 1165 & 53571.047 & 232 & \pm 178 & \\
\hline ALS 9313 & LS IV -12 1 & SD:O & 60000 & 4.5 & 2760 & 1000 & 53566.068 & 207 & \pm 239 & \\
\hline HD 149382 & BD-03 3967 & SD:OB & 34200 & 5.9 & 696 & 490 & 53458.390 & -268 & \pm 731 & \\
\hline HD 171858 & CD-23 14565 & SD:B & & & 876 & 1945 & 53512.357 & -32 & \pm 136 & \\
\hline CD-51 11879 & LSE 263 & SD:O & 70000 & 4.9 & 2766 & 1270 & 53512.395 & 392 & \pm 262 & \\
\hline HD 188112 & CD-28 16258 & $\mathrm{SD}: \mathrm{B}+?$ & 21500 & 5.7 & 1170 & 470 & 53565.291 & -228 & \pm 690 & \\
\hline CD-23 15853 & LSE 21 & SD:O & 100000 & & 2580 & 1020 & 53533.347 & -332 & \pm 561 & \\
\hline HD 205805 & CD-46 14026 & SD:B & 25000 & 5.0 & 1152 & 1810 & 53533.384 & -139 & \pm 120 & \\
\hline JL 87 & EC 21435-7634 & SD:B & 28000 & 5.2 & 2760 & 950 & 53597.196 & -64 & \pm 153 & \\
\hline WD $2148+286$ & $\mathrm{BD}+284211$ & SD:O & & & 1572 & 1240 & 53533.414 & -121 & \pm 415 & \\
\hline CD-35 15910 & SB 814 & SD:B & 28800 & 5.4 & 4800 & 1385 & 53598.378 & 246 & \pm 232 & \\
\hline TD1 32702 & [CW83] 0512-08 & SD:B & 38000 & 5.6 & 2640 & 1600 & 53058.025 & 209 & \pm 160 & $*$ \\
\hline CPD-64 481 & PPM 354969 & SD:B & 27500 & 5.0 & 2640 & 1350 & 53058.069 & 100 & \pm 230 & $*$ \\
\hline CD-31 4800 & ALS 591 & SD:O & 44000 & 5.4 & 1440 & 1565 & 53058.215 & 90 & \pm 140 & $*$ \\
\hline HD 76431 & $\mathrm{BD}+022100$ & SD:B & 31000 & 4.5 & 1800 & 2675 & 53058.255 & 38 & \pm 76 & $*$ \\
\hline PG 0909+275 & PG 0909+276 & SD:B & 35400 & 6.0 & 7200 & 1345 & 53058.139 & -23 & \pm 194 & $*$ \\
\hline CD-22 9142 & EC 11481-2303 & SD:O & 42000 & 5.8 & 1510 & 1236 & 53134.112 & 636 & \pm 416 & ** \\
\hline & & & & & 1510 & 848 & 53144.110 & 348 & \pm 708 & ** \\
\hline CD-46 8926 & LSE 153 & SD:O & 70000 & 4.8 & 9900 & 2275 & 53058.347 & -68 & \pm 158 & $*$ \\
\hline
\end{tabular}

Notes. In the last column, one asterisk $(*)$ means that the observations were previously published by O'Toole et al. (2005); two asterisks $(* *)$, that the data were published by Jordan et al. (2007).

None of the 26 field measurements of 24 different sdB and sdO stars shows any significant detection. (Although two measurements, of HD 4539 and PG $0133+144$, have $\left\langle B_{z}\right\rangle$ values that differ from zero by somewhat more than $2 \sigma$, this does not strongly suggest the presence of fields, as Bagnulo et al. 2012 have shown that even with the new reductions, standard errors of FORS1 field measurements tend to be somewhat underestimated.) This null result is confirmed with other reasonable choices for reduction flags, which (as discussed by Bagnulo et al. 2012) change the final field values from the values tabulated here by typically $1 \sigma$. Four measurements reported in Table 1 are reobservations of previously observed hot subdwarfs. In two cases the initial report of non-detection of a field, of HD 149382 (Borra et al. 1983) and of SB 815 (Mathys et al. 2012), is confirmed by our non-detection; and our two non-detections of a field in SB 290, together with two non-detections in three measurements in the initial study by Mathys et al. (2012), suggest that the single marginal detection reported of this star is probably not real.

One of the original aims of these observations was to investigate the magnetic properties of hot subdwarfs from different populations (He-rich or in binaries for example); however, the non-detections reported here suggest that if there are differences, they are too small for us to currently detect.

\section{Discussion and conclusions}

Considering our own data in Table 1, together with the measurements reported in the literature, we conclude that there are no secure detections of magnetic fields in hot subdwarf stars yet, although at least three or four hot subdwarfs have reported fields that may be shown on further study to be real. Looking at the more than twenty stars discussed here, for which field measurements have failed to reveal any field with standard errors of the order of 2-400 G, we conclude that globally dipolar fields of $\mathrm{kG}$ strength occur in at most a few percent of hot subdwarfs. Our data are consistent with the possibility that fields so large may be completely absent from the hot subdwarfs. This result suggests that the most valuable kind of further observational survey would be one which reaches the smallest meaurement uncertainty possible for a significant number of hot subdwarfs.

The apparent magnitudes of hot subdwarfs, and the richness of their spectra, varies greatly from one star to another, but many are suitable for field measurements with uncertainties of the order of a hundred $\mathrm{G}$ or even a few tens of $\mathrm{G}$. This precision can be reached with a variety of spectropolarimeters, including some of those discussed in Sect. 2. We believe that it is quite important to carry out substantial further surveys for hot subdwarf fields, similar to the ones carried out by O'Toole et al. (2005) and Mathys et al. (2012), and particularly to re-observe hot subdwarfs for which field detections have been reported and not later shown to be spurious.

Such surveys of the brighter hot subdwarfs, especially those with numerous fairly sharp spectral lines, are best carried out on a high-resolution spectropolarimeter on intermediate-size telescopes, such as ESPaDOnS at CFHT, Narval at the Observatoire du Pic du Midi, or HARPSPol at ESO, La Silla. However, for fainter objects or for ones whose spectra are dominated by broad lines (e.g. Balmer lines), low-resolution spectropolarimeters, especially those on large-aperture telescopes, such as ESO's FORS2, the UAGS spectropolarimeter at the Russian 6-m telescope, or the Steward Observatory instrument have an important and valuable role to play. 
J. D. Landstreet et al.: The magnetic fields of hot subdwarf stars

Acknowledgements. J.D.L. acknowledges financial support from the Natural Sciences and Engineering Research Council of Canada.

\section{References}

Bagnulo, S., Landolfi, M., Landstreet, J. D., et al. 2009, PASP, 121, 993 Bagnulo, S., Landstreet, J. D., Fossati, L., \& Kochukhov, O. 2012, A\&A, 538, A129

Borra, E. F., Landstreet, J. D., \& Thompson, I. 1983, ApJS, 53, 151

Elkin, V. G. 1996, A\&A, 312, L5

Jordan, S., Aznar Cuadrado, R., Napiwotzki, R., Schmid, H. M., \& Solanki, S. K. 2007, A\&A, 462, 1097
Jordan, S., et al. 2012, A\&A, submitted

Kawka, A., Vennes, S., Schmidt, G. D., Wickramasinghe, D. T., \& Koch, R. 2007, ApJ, 654, 499

Landstreet, J. D. 2004, in The A-Star Puzzle, ed. J. Zverko, J. Ziznovsky, S. J. Adelman, \& W. W. Weiss, IAU Symp., 224, 423

Mathys, G., Hubrig, S., Mason, E., et al. 2012, Astron. Nachr., 333, 30

Østensen, R. H., Oreiro, R., Solheim, J.-E., et al. 2010, A\&A, 513, A6

O’Toole, S. J., Jordan, S., Friedrich, S., \& Heber, U. 2005, A\&A, 437, 227

Petit, P., Van Grootel, V., Bagnulo, S., et al. 2012, ASPC, 452, 87

Savanov, I. S., Romanyuk, I. I., Semenko, E. A., \& Dmitrienko, E. S. 2011, Astron. Rep., 55, 1115

Valyavin, G., Bagnulo, S., Fabrika, S., et al. 2006, ApJ, 648, 559 